\title{
Article \\ An Augmented Warning System for Pedestrians: User Interface Design and Algorithm Development
}

\author{
Yourui Tong $\mathbb{(}$, Bochen Jia $* \mathbb{D}$ and Shan Bao \\ Industrial and Manufacturing Systems Engineering, University of Michigan-Dearborn, Dearborn, MI 48127, USA; \\ tongy@umich.edu (Y.T.); shanbao@umich.edu (S.B.) \\ * Correspondence: bochenj@umich.edu
}

Citation: Tong, Y.; Jia, B.; Bao, S. An Augmented Warning System for Pedestrians: User Interface Design and Algorithm Development. Appl. Sci. 2021, 11, 7197. https://doi.org/ 10.3390/app11167197

Academic Editor: Marco Guerrieri

Received: 26 June 2021

Accepted: 2 August 2021

Published: 4 August 2021

Publisher's Note: MDPI stays neutral with regard to jurisdictional claims in published maps and institutional affiliations.

Copyright: (c) 2021 by the authors. Licensee MDPI, Basel, Switzerland. This article is an open access article distributed under the terms and conditions of the Creative Commons Attribution (CC BY) license (https:/ / creativecommons.org/licenses/by/ $4.0 /)$.
Featured Application: This study designed a warning system for pedestrians with an obstructed view or low situation awareness. Furthermore, it can extend into the autonomous driving environment, giving a warning to a pedestrian when a malfunction happens inside an autonomous vehicle.

Abstract: Warning pedestrians of oncoming vehicles is critical to improving pedestrian safety. Due to the limitations of a pedestrian's carrying capacity, it is crucial to find an effective solution to provide warnings to pedestrians in real-time. Limited numbers of studies focused on warning pedestrians of oncoming vehicles. Few studies focused on developing visual warning systems for pedestrians through wearable devices. In this study, various real-time projection algorithms were developed to provide accurate warning information in a timely way. A pilot study was completed to test the algorithm and the user interface design. The projection algorithms can update the warning information and correctly fit it into an easy-to-understand interface. By using this system, timely warning information can be sent to those pedestrians who have lower situational awareness or obstructed view to protect them from potential collisions. It can work well when the sightline is blocked by obstructions.

Keywords: augmented reality; graphical user interface; pedestrian safety; warning design

\section{Introduction}

Compared to vehicle drivers, other road users such as pedestrians or cyclists are more vulnerable when sharing the road with vehicles. Each year, 1.35 million fatalities and 20-50 million injuries worldwide are reported due to traffic accidents [1], and the overall number increases yearly [2]. Among these injuries and deaths, the rate of pedestrian deaths remains high and keeps rising [3], which can be linked to many moderating factors. For example, pedestrian injuries due to phone distraction increased more than three times from 2007 to 2010 [4]. Further, decreasing situational awareness could also lead to a pedestrian engaging in risky behavior during road sharing [5]. Due to the limited sensor accuracy and reliability, the rapid implementation of autonomous vehicles may lead to even higher pedestrian casualties [6]. It is necessary to develop a solution to reduce the potential threats from these known factors, especially when the pedestrian's view is blocked by a truck, bus, or building or when the pedestrian's situation awareness is low due to fatigue, distraction, etc. Due to such visual blockages, it could be very beneficial to have a visual-based warning system to make up for the information missed by the pedestrians. As a warning system, the information must be carried out in real-time. Moreover, due to the limitation of pedestrian's capacity, the warning system must be light in weight and easy to carry while the pedestrian is walking in public.

Different methods were developed to improve pedestrian safety and warn road users, though most of them focused on detecting the collision rather than warning the pedestrian 
of the potential risks [7]. Limited methods were developed to carry out warning pedestrians when a potential collision was detected. Smartphone audio [8] and vibration [9] were used to provide warning information to pedestrian users, however, they could not provide the multimodal cues as a graphically based information system can [10]. Dhondge [8] proposed a phone-based visual warning system for distracted pedestrians at intersections. Such a system, however, requires real-time interaction between the pedestrian and the mobile phone to deliver the warning message.

A visual system could present the warning information more directly and comprehensively compared with auditory- and tactile-based systems, which made it the best media to deliver an immediate warning to the pedestrians while giving them more time to respond [11]. However, pedestrians have limited carrying capability during walking, which limits the types of visual systems they can carry. It is also not safe to forcibly shift the pedestrian's attention from surrounding traffic to the visual system while sharing the road with other road users. Due to these limitations of pedestrians, traditional visualbased warning system designs using a large screen or a mobile phone are not feasible for pedestrians.

Augmented reality (AR) is a state-of-the-art technology that can "augment" the real world with virtual information so that users can perceive the augmented virtual information without losing their sense of the surrounding environment. Current devices that can display AR information include eyeglasses [12], heads-up displays (HUD) [13], contact lenses [14], handheld devices (e.g., smartphones) [15], etc. Since some of these AR devices could be carried with minimal effort (e.g., eyeglasses), they could be used as a feasible choice to present a visual warning system for pedestrians.

AR technologies are applied in the design of vehicle-based warning systems. Kim [16] developed an in-vehicle HUD user interface for crash warning systems. Plavsic [17] evaluated different AR warning systems for the driver in an urban environment. Among these existing studies of warning system design, the research mainly focused on warning drivers rather than pedestrians. In addition, most of these existing studies rely on a mounted HUD rather than on a wearable display. Such mounted displays could not be transferred to pedestrian application.

Handheld devices such as smartphones are smaller, portable, and could support touch screen inputs [14], which are more commonly used for the scenarios when mounted HUD is not available. However, it is infeasible for pedestrians to hold their smartphones while walking in public constantly. Since the warning needs to be carried out promptly, it is infeasible to design a system that requires the pedestrian to check on their phone even when they are not holding it to receive the warning information.

Compared with HUD devices and handheld deceives, wearable AR devices are usually lightweight enough for pedestrians to use without interrupting their normal walking. By using wearable AR devices, pedestrians can perceive both warning information and realworld information without taking their eyes off the road for long. As a common wearable AR device, AR glasses may cause the least interruption for pedestrians during walking. Although HoloLens, the platform used in this paper, was still too bulky and heavy to wear daily, the fast evolution of the AR glasses could lead to the development of future wearable AR solutions. For example, a newer version of the AR glasses, Glass Enterprise Edition 2, was only $46 \mathrm{~g}$ and was designed to wear all day [18]. Overall, AR glasses are lightweight, can constantly be worn, and there is no need to hold them in hand or pay constant attention as with other AR devices. These potential advantages of AR glasses make them a perfect candidate to carry out the warning information for pedestrians in public with minimal interference. However, the visual field of wearable AR devices is usually superimposed at the center of the user's field of view all the time, which may cause extra distraction or block the user's view. Therefore, it is important to ensure that such AR devices activate the warning interface only when needed. To the authors' knowledge, no research was done to develop an augmented visual-based warning system to deliver warning information about potential risks to pedestrians in real-time. 
Therefore, the objective of this study was to develop an AR-based wearable interface to deliver warning information to pedestrians after a potential collision is detected. A HoloLens setup was used as an experimental platform to develop the user interface, and a series of projection algorithms were used to carry out the warning information in real-time.

\section{Materials and Methods}

There are several commercial AR glasses in the market, for example, Microsoft HoloLens and Sony smart glasses. In this study, AR structure and parameters from the Microsoft HoloLens were used to design the warning system to deliver location-based warning information. To ensure that the interface can provide accurate warning information in a timely way, a series of projection algorithms were developed to provide real-time updates of the warning information based on the spatial data collected from the vehicle and the pedestrian. As shown in Figure 1, series of data were required from the vehicle and the pedestrian to identify vehicle position, pedestrian position, and distance between the vehicle and the pedestrian. The corresponding warning information was then generated and presented to the pedestrian through a wearable AR device. Different projection algorithms were used for different vehicle-pedestrian interaction scenarios.

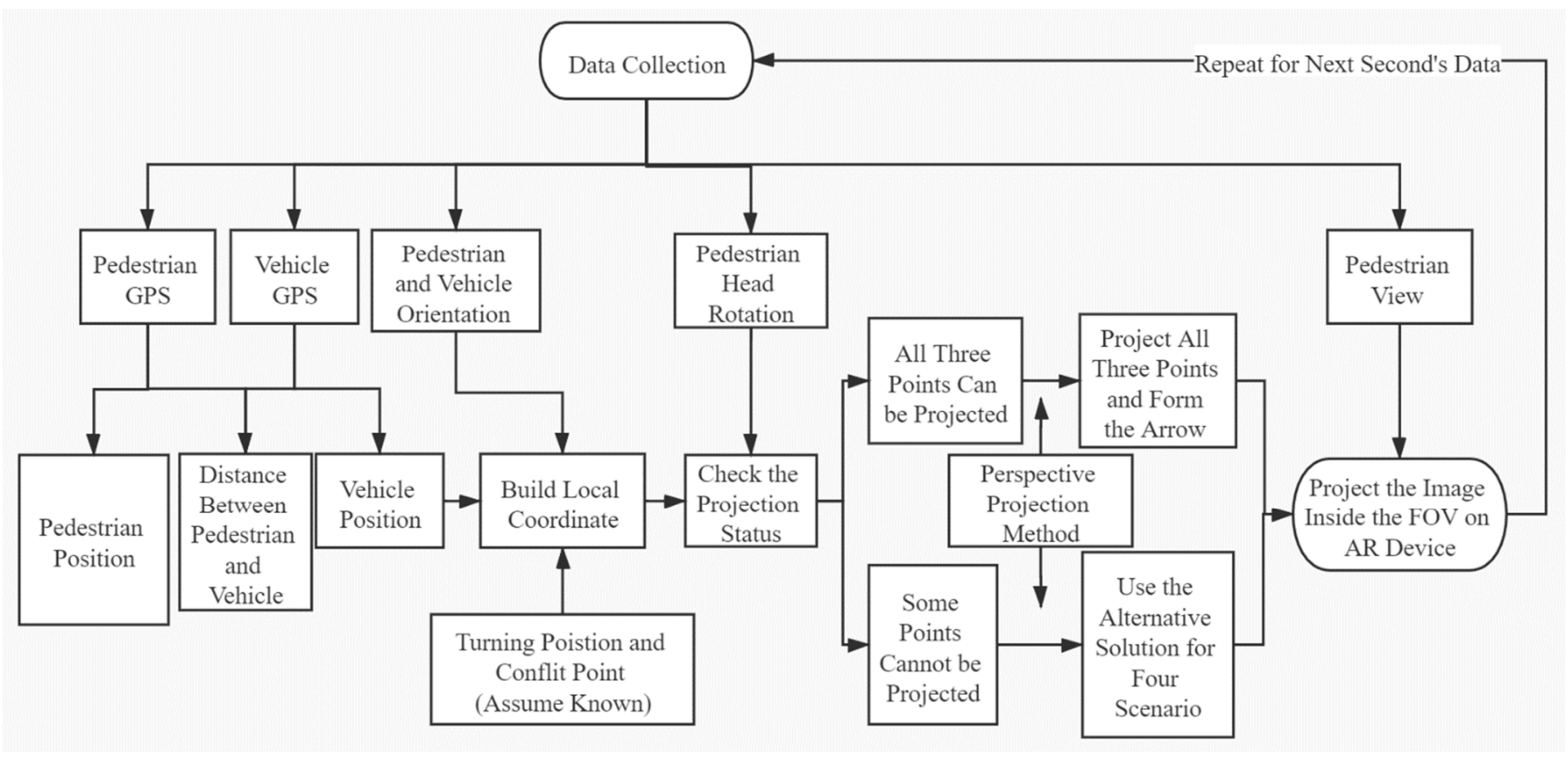

Figure 1. Warning system summary.

\subsection{Interface Development}

Since the AR glasses can be worn by a pedestrian during normal walking in public, it is important to deactivate the warning system while no conflict is detected. Moreover, the AR interface should not be so complicated [19] that it interrupts the pedestrians perceiving the real-world information. Redundant information in the interface may increase the mental workload and the response time for the pedestrians when perceiving the warnings [20]. Thus, only necessary information should be shown in the warning interface. Since the warning system is designed for the pedestrian when their view is obstructed or situation awareness is low, the information the pedestrian cannot perceive, such as the direction and the expected path of the oncoming vehicle, the conflict point, as well as the time to the collision, should be shown to the pedestrian. With such information present, the pedestrian is able to identify the location of the oncoming vehicle and the level of the risk. Since there are no well-built guidelines for the AR interface design, the smartphone and the human-computer interface designs were used to match the top-down human perception 
process [21]. A sidebar design was used to show the oncoming vehicle direction based on smartphone and human-computer interface designs [22,23]. Moreover, general warning information design rules, such as color code [24] or a warning symbol, were adapted in the current design of the warning information to match the existing mental models for the general public $[25,26]$. Further details about interface design can be found in the previous study [27].

Derived from the previous research [27], the warning window was defined as a $60^{\circ} \times 30^{\circ}$ rectangular area on the projection plane, where the center of this rectangle was located at the center of the pedestrian's sight. AR information was projected on the virtual projection plane in front of the eyes. The distance between the eyes and the projection plane was defined as the projected distance. According to the design of the HoloLens AR display, the optimal projected distance was $2 \mathrm{~m}$, which minimized the discomfort from the vergence-accommodation conflict and ensured the optimal overlap of the two display lenses that a HoloLens has [28]. Based on the projection rules, the warning window size was proportionally adjusted to $2.31 \mathrm{~m}$ and $1.16 \mathrm{~m}$ from $2 \mathrm{~m}$ away.

After the size and the position of the warning window were defined, the detailed components inside the warning window were designed. The vehicle location, the expected vehicle path, the expected conflict point, the collision time, the warning symbol, and the attention-leading component (i.e., a sidebar and an arrow to attract and lead the pedestrian's attention to the hazard) were included in the warning interface, derived from the previous study [27]. Figure 2 shows the layout of all these components presented in the warning window.

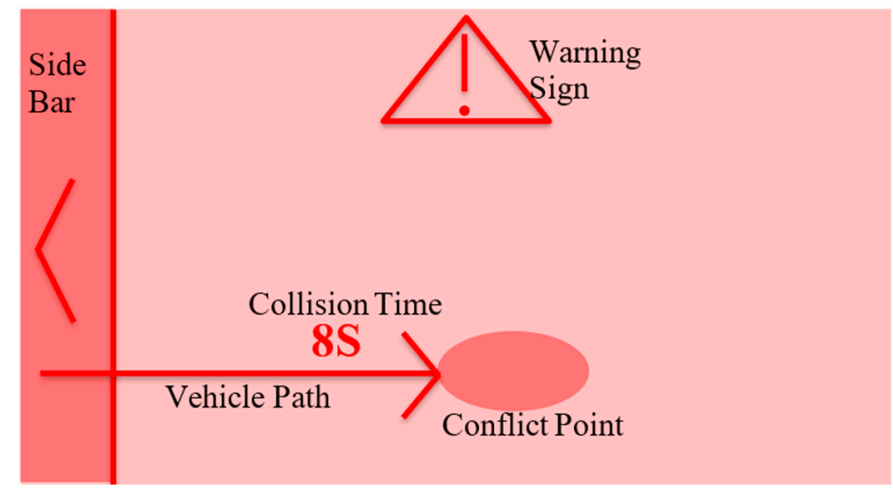

Figure 2. Components in the warning window.

Figure 3 shows an example of the user interface overlaid on the real-world view in which a vehicle was coming from the left and crossing straight in front of the pedestrian.

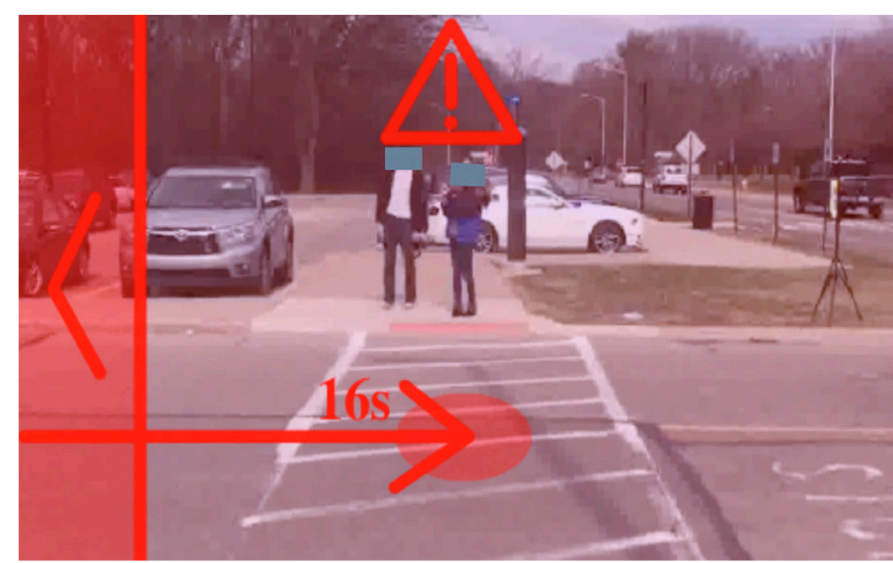

Figure 3. User interface overlapped with a real-world view. 
As shown in Figure 3, an arrow sign was used to indicate that a vehicle was coming from the left side of the road. The location of the sidebar matched the location of the oncoming vehicle, and the arrow sign on the sidebar indicated that more information was available along the arrow direction. In this example, the sidebar with the left arrow on the left side of the warning window indicated that the upcoming vehicle was coming from the left side and could be seen by turning the head to the left. The conflicting time, $16 \mathrm{~s}$, indicated that the vehicle was due to reach the red dot position in $16 \mathrm{~s}$. The sidebar disappeared after the conflicting vehicle appeared in the warning window. The arrow position and the conflicting time updated in real-time based on the pedestrian heading direction. The related warning information was updated (i.e., appeared or disappeared) based on pedestrian instant facing direction.

\subsection{Projection Algorithms Development}

To ensure that the interface provided instant warning information to the pedestrian based on his/her location, an algorithm was developed to determine the projection of the vehicle's path on the AR interface based on the instant pedestrian position. Global positioning system (GPS) data and inertial measurement unit (IMU) data from the vehicle were used to determine the vehicle pathway using the perspective projection method [29].

The GPS and the IMU from AR glasses can be used to track the pedestrian's instant location and their head rotation/facing direction, respectively. It is assumed that the pedestrian does not look to the side without rotating their head, thus the pedestrian's head rotation can be used to represent their viewing direction. This system would be activated after the potential conflict is detected and the conflict point is identified.

The algorithms for conflict detection and pedestrian/vehicle positioning are not introduced here because they are beyond the scope of this study. The focus of this study was developing algorithms and interfaces to correctly and efficiently present relevant warning information on the AR display. Overall, the data used in the system included vehicle position and orientation, pedestrian's position and orientation, pedestrian's facing direction, predicted conflict position if no action was taken, and predicted turning position. Predicted conflict position and predicted turning position were assumed already known from conflict detecting algorithms. In detail, a two-step algorithm was developed to first convert and synchronize the vehicle position, the conflict position, as well as the vehicle turning position into the pedestrian's local coordinates and further project the localized information on the projection plane using perspective projection methods.

The local coordinates used the pedestrian's position as the origin $(0,0)$, the pedestrian's facing direction as $+z$, and the pedestrian's right-hand direction as $+x$. After the coordinates' transformation, the warning information needed to be projected on the projection plane. Different methods can be used to project the 3D world onto a 2D plane. However, many of these methods may lose the depth cue during the projection and confuse the audience when they read the projected information. For example, the orthographic projection method uses orthogonal projection lines to project 3D items onto a 2D plan [30], which means the projected image on the $2 \mathrm{D}$ plane is the same size no matter the distance of the $3 \mathrm{D}$ item from the 2D plane. However, the human visual system needs such a depth cue to understand the distance between objects. It can confuse the audience if the depth cue is lost during the projection. In this study, the depth cue was necessary for the projection not to lose the accuracy of the warning information. The weak perspective projection method uses a similar concept to the orthographic projection methods [31], thus it loses the depth cue when doing the projection as well and thus also could not be used in this study. Perspective projection, on the other hand, is a method that can project a 3D object onto a 2D plane and not lose the depth cue [29] because the projection line it uses is the line connecting the 3D item to human eyes rather than orthogonal projection lines. Thus, the perspective projection method was used in this study to do the projection. The basic perspective projection matrix is shown in Equation (1) [32]: 


$$
\left[\begin{array}{llll}
1 & 0 & 0 & 0 \\
0 & 1 & 0 & 0 \\
0 & 0 & 1 & 0 \\
0 & 0 & \frac{1}{d} & 0
\end{array}\right] \times\left[\begin{array}{l}
x \\
y \\
z \\
1
\end{array}\right]=\left[\begin{array}{c}
x \\
y \\
z \\
z \\
d
\end{array}\right]
$$

The $\left[\begin{array}{l}x \\ y \\ z\end{array}\right]$ represents the $3 \mathrm{D}$ coordinates of a point needing to be projected, and $\mathrm{d}$ is the distance from the point to the projecting plane. Here, $(x, y, z)$ represents the transformed $3 \mathrm{D}$ position of pedestrian, vehicle, and conflict point in the pedestrian's local coordinates. In summary, the pedestrian's 3D local coordinates use the pedestrian's head location as origin; positive $x$ represents the direction toward the right lateral of the pedestrian, positive $y$ represents the pedestrian's cranial direction, and $\mathrm{z}$ represents the pedestrian's facing direction. All positions mentioned above were projected to the projection plane by using Equation (1). The location of the projected point on the projection plane was $(x \times d / z$, $y \times d / z)$. However, the coordinates needed to be updated in real-time based on where the pedestrian was looking so that the projection could have an accurate rotation angle. As mentioned above, it was assumed that pedestrians do not look to either side without turning their heads. Thus, a real-time coordinates rotation was needed for the projection matrix to cooperate with the pedestrian's head rotation so that the projection could be updated in real-time. After using a rotation matrix on $\left[\begin{array}{l}x \\ y \\ z\end{array}\right],\left[\begin{array}{l}C_{x} \\ C_{y} \\ C_{z}\end{array}\right]$ could be found for the point that needed to be projected. Then, the projection matrix could be used as follows:

$$
\left[\begin{array}{cccc}
1 & 0 & 0 & 0 \\
0 & 1 & 0 & 0 \\
0 & 0 & 1 & 0 \\
0 & 0 & \frac{1}{d} & 0
\end{array}\right] \times\left[\begin{array}{c}
\mathrm{C}_{\mathrm{x}} \\
\mathrm{C}_{\mathrm{y}} \\
\mathrm{C}_{\mathrm{z}} \\
1
\end{array}\right]=\left[\begin{array}{c}
\mathrm{P}_{\mathrm{x}} \\
\mathrm{P}_{\mathrm{y}} \\
\mathrm{P}_{\mathrm{z}} \\
\mathrm{P}_{\mathrm{w}}
\end{array}\right]
$$

$$
\left[\begin{array}{l}
P_{x} \\
P_{y} \\
P_{z} \\
P_{w}
\end{array}\right] \text { is the vector calculated after the perspective projection matrix was performed. }
$$

As mentioned in Equation (2), the projected vehicle point's coordinates located on the projection plane should be $\left(\mathrm{P}_{\mathrm{x}} / \mathrm{P}_{\mathrm{w}}, \mathrm{P}_{\mathrm{y}} / \mathrm{P}_{\mathrm{w}}\right)$. The perspective projection method was also used to project the conflict position. After projecting these two positions on the projection plane, the vehicle's path could be formed by connecting the vehicle point and the conflict point. Figure 4 shows an example of forming the vehicle path based on the projected vehicle and conflict points.

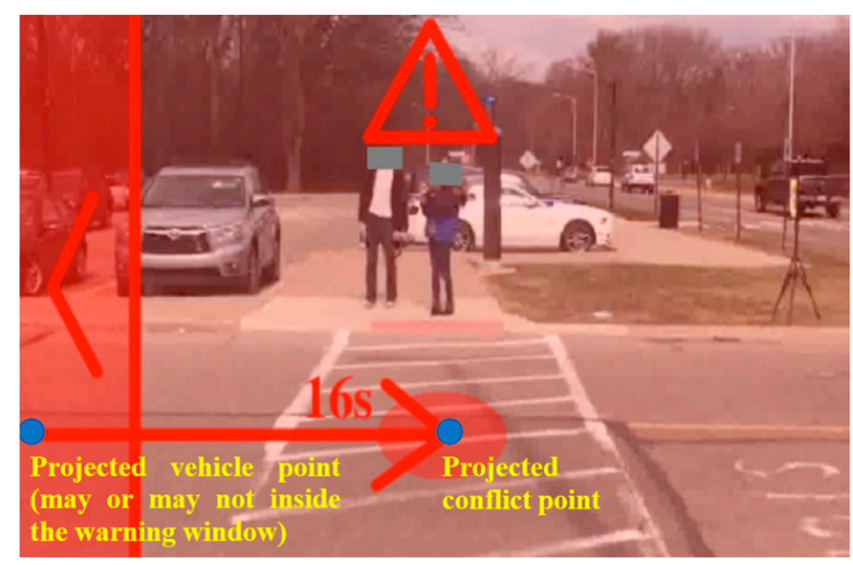

Figure 4. An example of forming an arrow based on projected points. 
A vehicle turning situation is a special situation that needed to be considered. To form the vehicle turning path, the vehicle turning position needed to be converted into the pedestrian's local coordinates and then projected to the projection plane using the same methods mentioned earlier. The projection methods may not always be able to project all the points within the warning window size based on the vehicle's and the pedestrian's instant locations. In this study, five specific scenarios were studied. One basic scenario was when the vehicle, the vehicle turning position, and the conflict point were all in front of the projection plane and could be projected inside the warning window. The vehicle path could be formed by simply connecting all three projected points. Figure 5 shows an example of the basic scenario.

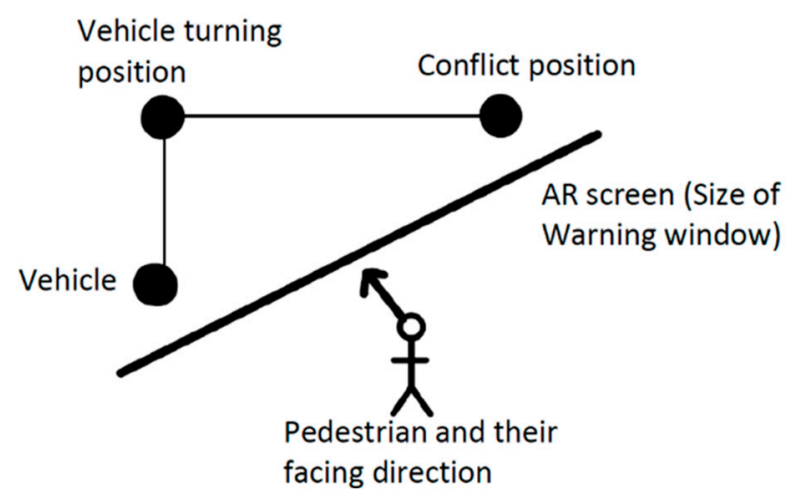

Figure 5. Basic turning scenario.

However, there were four other scenarios in which a simple connection of the points was not feasible, i.e., not all the points could be projected on the projection plane, as shown in Figure 6.
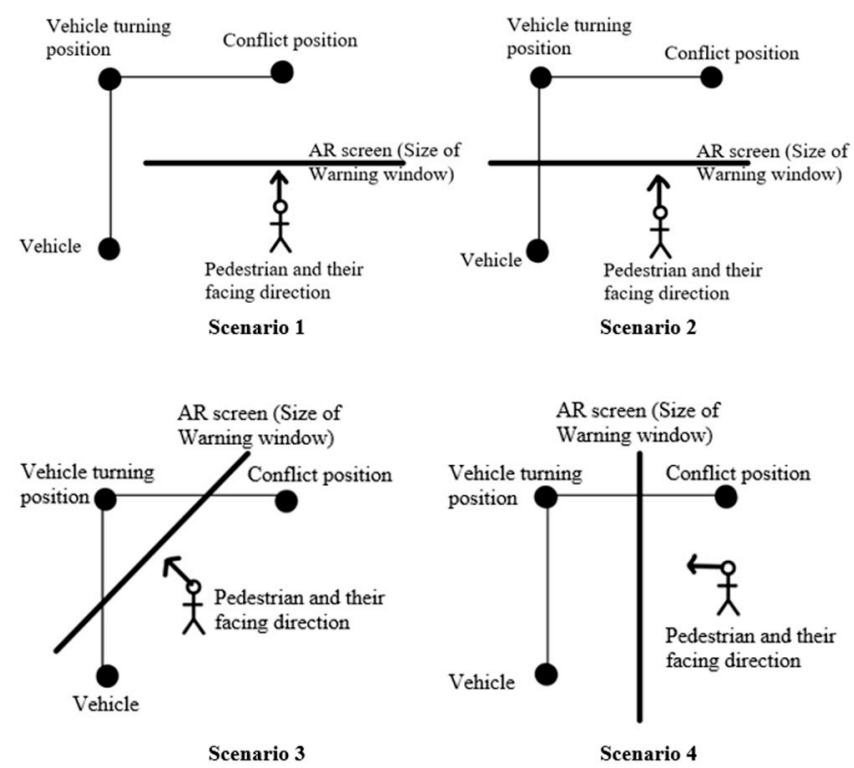

Figure 6. Four scenarios in which perspective projection did not work well.

In scenario 1, the vehicle turning position and the conflict position were in front of the projection plane, while the vehicle location was behind the projection plane, and the projected vehicle turning point was outside the warning window. In this scenario, the vehicle position was not projectable to the projection plane since it was behind the plane, and the projected vehicle turning point was outside the warning window, which made it infeasible to simply connect the projected points to show the vehicle path. To solve this problem, an adjusted turning point was used. The original projected vehicle turning point 
and the projected conflict point were connected to form a line, and the adjusted turning point was found by using the intersecting point of this line and the warning window boundary line. This adjusted turning point was used to ensure the vehicle turning point inside the warning window. The vehicle position in this scenario was represented using one of the corners of the warning window based on the vehicle position (e.g., the lower-left corner for vehicles coming from the left side behind the pedestrian). The adjusted turning point, the projected conflict point, and one of the warning window corners were used to form the vehicle path arrow. Figure 7 shows an example of scenario 1.

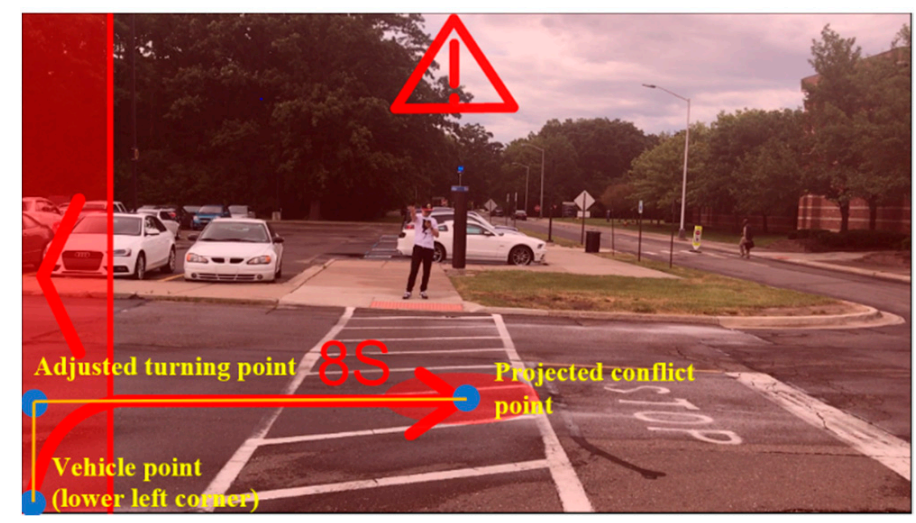

Figure 7. Example of scenario 1: Turning point and vehicle point could not be projected.

As shown in Figure 8, scenario 2 presents the situation in which the vehicle turning position and the conflict position were in front of the projection plane, and their projected points were both inside the warning window. However, the vehicle position was behind the projection plane and was not able to be projected on the plane. Similar to scenario 1, one corner of the warning window was used as the vehicle point based on the vehicle's position.

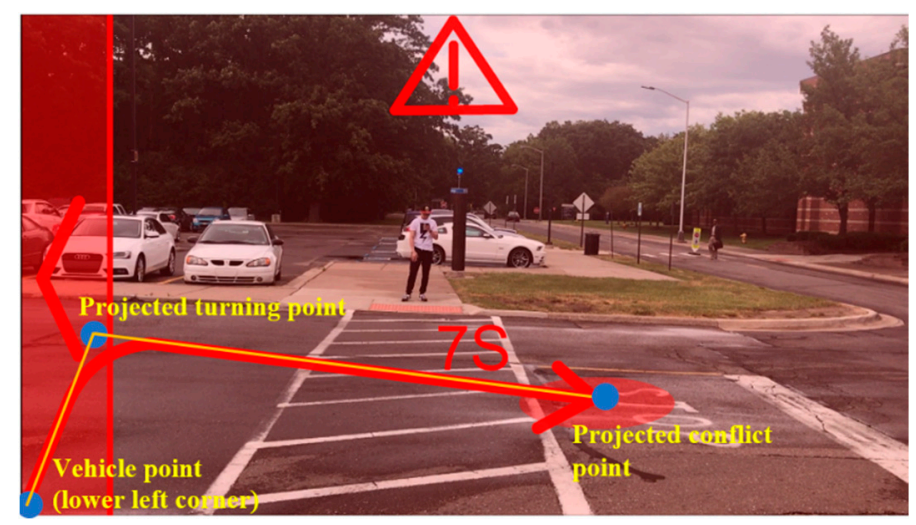

Figure 8. Example of scenario 2: Vehicle point could not be projected.

Scenario 3 described the situation in which only the vehicle turning position was in front of the projection plane and was able to be projected. As shown in Figure 9, a curved arrow centrally anchored at the projected vehicle turning point was generated to illustrate the direction of the upcoming vehicle.

Scenario 4 presented a situation in which the conflict position was behind the projection plane. Only the vehicle's heading direction was used to show the vehicle's path for such a situation. Two consecutive vehicle points were used to project two vehicle points on the projection plane, and the line connecting these two points was extended toward the direction the vehicle was heading to form the vehicle path. Figure 10 shows an example of scenario 4. 


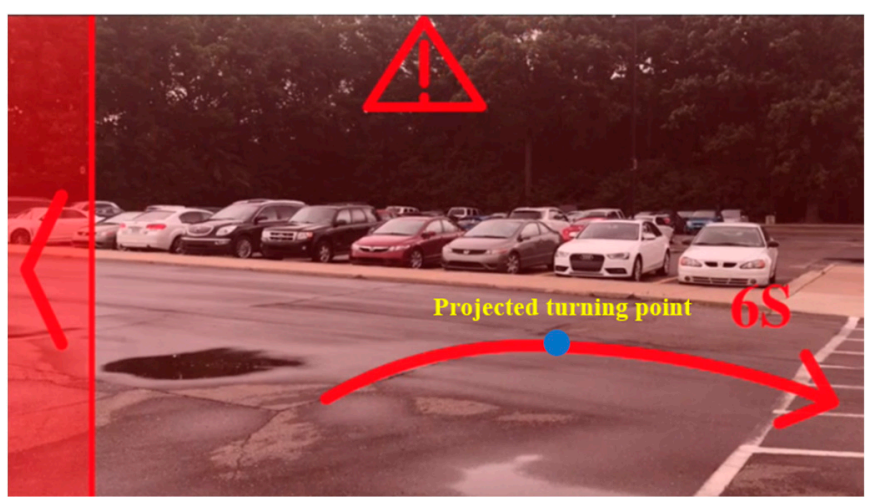

Figure 9. Example of scenario 3: Only the turning point could be projected in the warning window.

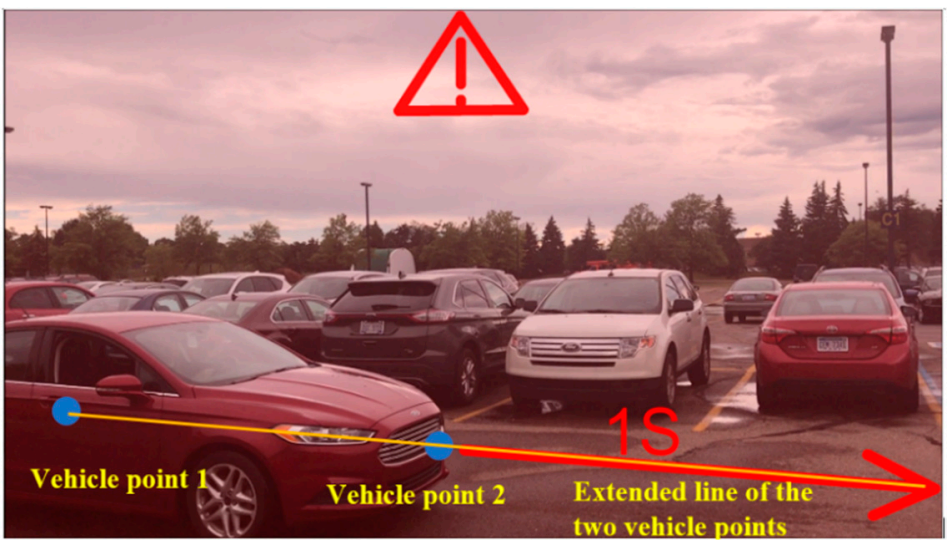

Figure 10. Example of scenario 4: Conflict position could not be projected.

\subsection{Evaluation}

The interface was evaluated in the previous study, and the results showed that the participants could understand the user interface well and could react correctly to avoid an accident [27]. To test the performance and the accuracy of the developed projection algorithm, two experimenters simulated a vehicle-pedestrian conflict scenario at a campus parking lot, and data from both simulated pedestrian and vehicle were collected to evaluate the performance of the developed algorithm. In detail, an experimenter simulating a pedestrian walked toward the footpath. The experimenter stopped when a signal was received and turned to look at the vehicle driven by another experimenter. A smartphone fixed at the experimenter's eye level was used to collect the pedestrian's GPS data, IMU data, and video of the instant view of the pedestrian. A vehicle was driven by another experimenter, and the vehicle data were collected using the same type of device fixed on the dashboard of the vehicle. The vehicle was located by the simulated pedestrian's left side of the road. When the simulated pedestrian approached the crosswalk, the vehicle received a signal and started driving toward the crosswalk to simulate the vehicle-pedestrian conflict.

The initial sampling rate of GPS and IMU data was $600 \mathrm{~Hz}$. Once the data were cleaned, GPS and IMU were down-sampled to $1 \mathrm{~Hz}$ to feed in the projection algorithm. As mentioned in Equation (2), the projected point $\left(\mathrm{P}_{\mathrm{x}} / \mathrm{P}_{\mathrm{w}}, \mathrm{P}_{\mathrm{y}} / \mathrm{P}_{\mathrm{w}}\right)$ was calculated for every input point. If the projected point was outside of the warning window, the intersection of the vehicle path and the warning window boundary was used as the projected point. An ideal vehicle path was visually identified and manually drawn on the video image based on the location of the vehicle and the direction of the road. To check the accuracy of the algorithm, the average distance between the projected points and the ideal vehicle path was calculated. The smaller the distance between the projected points and the ideal vehicle path was, the more accurate the algorithm was. The vehicle path was formed by connecting the vehicle point and the conflict point. The slope of the projected and the ideal 
vehicle path was calculated based on these two points to test the vehicle path direction. The closer the projected slope was to the ideal slope, the more accurate the vehicle path direction was.

\section{Result}

A total of $8 \mathrm{~s}$ of data were used to test the algorithm. The projected points inside the warning window were calculated for each second of the data. Table 1 shows the projected vehicle and conflict points inside the warning window. For each second, the x-axis and the $y$-axis locations for the points were calculated.

Table 1. Vehicle projection points and conflict projection points.

\begin{tabular}{ccccc}
\hline \multirow{2}{*}{ Time } & \multicolumn{2}{c}{ Vehicle Point } & \multicolumn{2}{c}{ Conflict Point } \\
\cline { 2 - 5 } & x-Axis & y-Axis & x-Axis & y-Axis \\
\hline $1 \mathrm{~s}$ & 0.000 & 0.542 & 1.155 & 0.460 \\
$2 \mathrm{~s}$ & -0.834 & 0.580 & 1.155 & 0.218 \\
$3 \mathrm{~s}$ & -1.155 & 0.523 & 1.155 & -0.078 \\
$4 \mathrm{~s}$ & -1.155 & 0.288 & 0.171 & -0.091 \\
$5 \mathrm{~s}$ & -1.155 & 0.255 & 0.089 & -0.107 \\
$6 \mathrm{~s}$ & -1.155 & 0.175 & -0.108 & -0.143 \\
$7 \mathrm{~s}$ & -1.155 & 0.063 & -0.355 & -0.194 \\
$8 \mathrm{~s}$ & -1.155 & 0.272 & 0.029 & -0.278 \\
\hline
\end{tabular}

Once the projected points were calculated, the vehicle path was formed and was overlaid on the video images based on the $x$ and the $y$ locations in the warning window. An ideal vehicle path was visually identified and manually drawn on the video image based on the location of the vehicle in the image and the direction of the road. Figure 11 shows an example of the projected vehicle path and the ideal vehicle path in the video at $1 \mathrm{~s}, 3 \mathrm{~s}, 5 \mathrm{~s}$, and $7 \mathrm{~s}$.

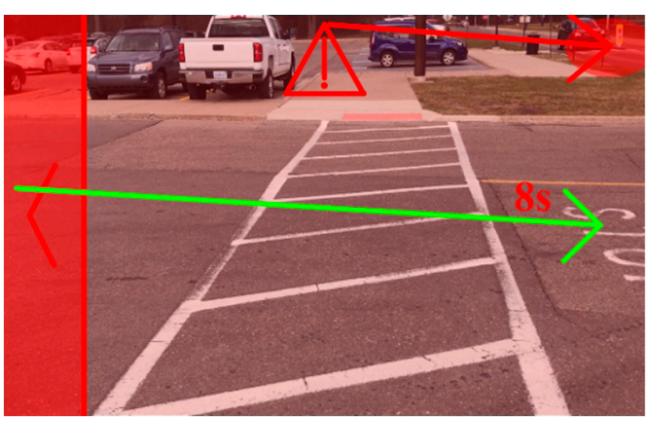

(a)

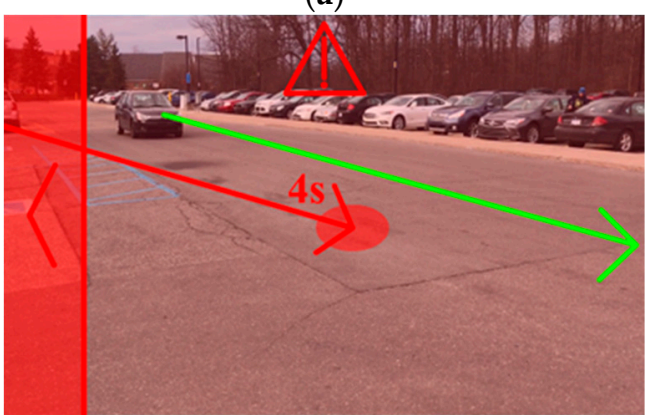

(c)

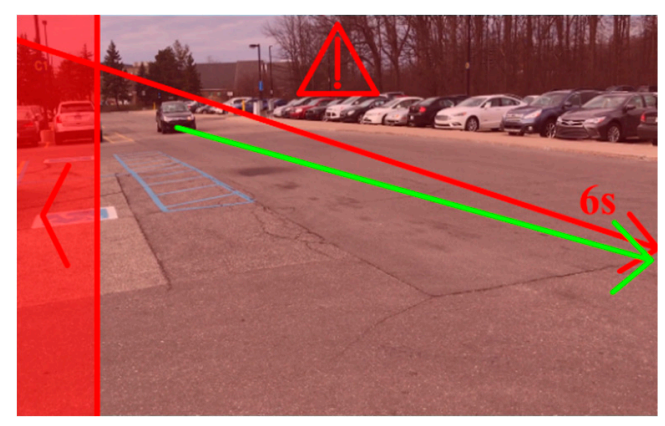

(b)

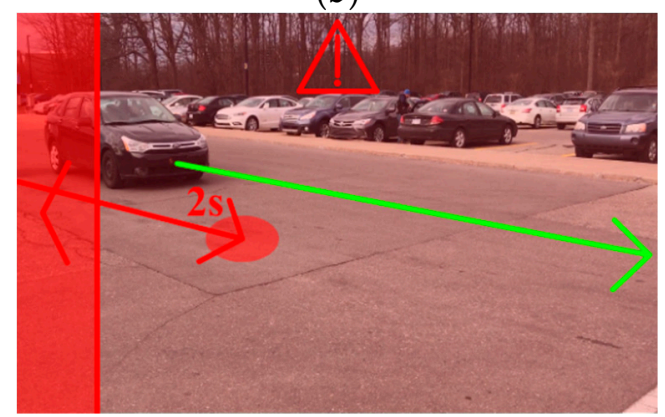

(d)

Figure 11. Projected video path (red) and ideal vehicle path (green) in the video image. (a) Vehicle path at $1 \mathrm{~s}$; (b) vehicle path at $3 \mathrm{~s}$; (c) vehicle path at $5 \mathrm{~s}$; (d) vehicle path at $7 \mathrm{~s}$. 
The distances from the projected vehicle and conflict points to the ideal path were calculated for each second of the data. The average of these two distances was calculated as well. An overall average among all $8 \mathrm{~s}$ of the data was then calculated. As shown in Table 2, two distances from the projected vehicle path to the ideal vehicle path and the average of these two distances were calculated. The overall average of the calculated distance was 0.253 .

Table 2. Distance between projected points and ideal points.

\begin{tabular}{cccc}
\hline Time & $\begin{array}{c}\text { Distance from Projected } \\
\text { Vehicle Points to the Ideal } \\
\text { Path }\end{array}$ & $\begin{array}{c}\text { Distance from Projected } \\
\text { Conflict Points to the Ideal } \\
\text { Path }\end{array}$ & \begin{tabular}{c} 
Average Distance \\
\hline $\mathrm{s}$
\end{tabular} $\mathrm{c}^{\mathrm{s}}$ \\
$3 \mathrm{~s}$ & 0.526 & 0.504 & 0.515 \\
$4 \mathrm{~s}$ & 0.418 & 0.315 & 0.367 \\
$5 \mathrm{~s}$ & 0.086 & 0.065 & 0.076 \\
$6 \mathrm{~s}$ & 0.142 & 0.188 & 0.165 \\
$7 \mathrm{~s}$ & 0.174 & 0.223 & 0.199 \\
$8 \mathrm{~s}$ & 0.227 & 0.292 & 0.259 \\
& 0.176 & 0.298 & 0.237 \\
& 0.088 & 0.333 & 0.210 \\
\hline
\end{tabular}

As described above, the slope of the vehicle path was calculated based on vehicle points and conflict points for both the projected vehicle path and the ideal vehicle path. The difference between the projected slope and the ideal slope was calculated to measure the accuracy of the projected vehicle path direction. Table 3 shows the result of the slope and the differences. Overall, both projected and ideal paths had slopes less than 0.3 . The largest slope was observed from the projected vehicle path at $8 \mathrm{~s}$, which also caused the largest slope difference between the projected and the ideal path.

Table 3. Differences between projected and ideal slopes.

\begin{tabular}{cccc}
\hline Time & Projected Slope & Ideal Slope & Difference \\
\hline $1 \mathrm{~s}$ & -0.071 & -0.052 & 0.019 \\
$2 \mathrm{~s}$ & -0.182 & -0.130 & 0.052 \\
$3 \mathrm{~s}$ & -0.260 & -0.251 & 0.009 \\
$4 \mathrm{~s}$ & -0.286 & -0.251 & 0.035 \\
$5 \mathrm{~s}$ & -0.291 & -0.251 & 0.040 \\
$6 \mathrm{~s}$ & -0.304 & -0.240 & 0.065 \\
$7 \mathrm{~s}$ & -0.322 & -0.167 & 0.154 \\
$8 \mathrm{~s}$ & -0.465 & -0.251 & 0.214 \\
\hline
\end{tabular}

\section{Discussion}

The purpose of this study was to design an AR-based system to carry visual warning information to pedestrians who are walking in a public area. The interface and the projection algorithm developed in this study show the possibility of providing real-time visual-based warning information to pedestrians using wearable devices. The warning information carried through the interface includes vehicle position, vehicle path, conflict point, collision time, and sidebar. Different pedestrian-vehicle interaction scenarios were studied, and corresponding algorithms were developed.

Overall, the developed interface and algorithms presented a reasonable performance that could be used to carry out timely warning information to the pedestrians. The interface was easy to understand for the majority of the participants with minimal learning requirements. All the participants were able to give correct reactions after seeing the warning information. In addition to the interface, the projection algorithm could project 
the vehicle path and show the warning information correctly in real time. As shown in Figure 11, only the first projected vehicle path from the beginning of the data collection was away from the ideal path; all other projected paths were closer to the ideal path and provided the correct vehicle information to the pedestrians. Although the first projected vehicle path was away from the ideal path, the slope of the projected path matched the ideal path, which meant, even though the projected path was not overlaid on the correct lane of the road, the vehicle direction was still projected correctly. The slopes between the projected path and the ideal path were compared, and the average difference was only 0.074, which was small enough to give the pedestrian correct information. Among all the data, the lowest difference between the ideal slope and the projected slope was 0.009 , and the largest difference was 0.214 . On average, the closer the vehicle was to the pedestrian, the higher was the observed difference in slope. When the vehicle was getting closer to the pedestrian, the distance between vehicle point and conflict point was shorter, and the slope difference got larger, even with a small mismatch between projected points and ideal points. It was acceptable when the slope difference was getting larger when the vehicle was getting closer to the pedestrians, since pedestrians could more easily identify the vehicle and the vehicle path regardless of the potential projection errors, as the vehicle was near the conflict point. The distances between the projected path and the ideal vehicle path were also compared. The average distance between projected points to the ideal vehicle path was 0.253 . Considering the overall size of the screen was 2.31 by 1.16 , the error of 0.253 was acceptable. In general, the error from slope was smaller, which meant the direction of the vehicle path was correct, and the distance between the two paths was larger, which meant the vehicle path may not have been overlaid on the exact lane in which the vehicle was coming. The errors may have been due to an error from the GPS signal. Better GPS hardware may be able to reduce such errors. Overall, the projection algorithms projected the information in the warning window correctly.

In summary, the developed warning system can clearly show the necessary information. As a system designed based on AR glasses, no extra carry effort is needed from pedestrians. The pedestrian still can see the real world while the warning system is on. Compared with a phone screen-based system [8], it could cause less distraction for pedestrians to receive real world information. Additionally, since it is a graphic warning system, pedestrians can receive more visual information compared with audio or vibration warning systems [9]. Such a system has the potential to deliver an effective warning to pedestrians who have lower situational awareness or are distracted and protect them from potential conflict. It can also give pedestrians information about an oncoming vehicle when the oncoming vehicle is blocked by other objects or buildings on the street. Moreover, this warning system can be applied in other situations. Since the input of this warning system only requires GPS and IMU of the vehicle and the pedestrian as well as the expected conflict location, it can integrate with any system that has such outputs. This design could be further extended into the autonomous driving environment, where it can give a warning to the pedestrian when there is some malfunction happening in an autonomous vehicle and the autonomous vehicles have potential to collide with the pedestrian.

A few limitations in this study should be acknowledged. First, the interface was not designed for situations in which multiple conflicts are detected. Future work could focus on the scalability of the designed system to include multiple conflicts simultaneously. Additionally, since current AR glasses are still too bulky and heavy to wear daily, the whole system is not supported by the existing hardware. In addition, current commercial AR glasses have a limitation on their field of view as well. The field of view of AR glasses is small; for example, the Microsoft HoloLens has only about a $35^{\circ}$ by $17^{\circ}$ field of view, and the Sony glasses are even smaller at $19^{\circ}$ by $6^{\circ}$ [33]. Thus, the designed size of the warning window, which is $60^{\circ}$ by $30^{\circ}$, cannot be fully shown in current AR glasses. Lastly, no quantitative evaluation was done for the user interface design. The reliability of the subjective evaluation is a concern, and objective measures could provide further information regarding the performance of the design. Objective evaluations, such as the 
subject's response time, accuracy, correct body physical response, etc., could be done further to quantify the effectiveness and the accuracy of the warning system.

Author Contributions: Conceptualization, Y.T. and B.J.; methodology, Y.T. and B.J.; software, Y.T.; validation, Y.T.; formal analysis, Y.T.; investigation, Y.T.; resources, B.J.; data curation, Y.T.; writingoriginal draft preparation, Y.T.; writing - review and editing, B.J. and S.B.; visualization, Y.T.; supervision, B.J.; project administration, B.J.; funding acquisition, B.J. All authors have read and agreed to the published version of the manuscript.

Funding: Research was funded by Michigan Mobility Transformation Center under grant number AWD001508.

Institutional Review Board Statement: Not applicable.

Informed Consent Statement: Not applicable.

Data Availability Statement: The data presented in this study are available on request from the corresponding author. The data are not publicly at this time due to sponsor agreement and as the data also forms part of an ongoing study.

Acknowledgments: The author would like to express very great appreciation to Sang-Hwan Kim and Di Ma from the University of Michigan-Dearborn, who provided insight and expertise that greatly assisted the research.

Conflicts of Interest: The authors declare no conflict of interest.

\section{References}

1. World Health Organization. Road Traffic Injuries. 27 April 2020. Available online: https://www.who.int/health-topics/roadsafety\#tab=tab_1 (accessed on 26 June 2021).

2. Peden, M.; Scurfield, R.; Sleet, D.; Mathers, C.; Jarawan, E.; Hyder, A.A.; Hyder, A.A.; Mohan, D.; Jarawan, E. World Report on Road Traffic Injury Prevention; World Health Organization: Geneva, Switzerland, 2004.

3. Retting, R.; Schwartz, S. Pedestrian Traffic Fatalities by State: 2017 Preliminary Data; Governors Highway Safety Association: Washington, DC, USA, 2017.

4. Basch, C.H.; Ethan, D.; Rajan, S.; Basch, C.E. Technology-related distracted walking behaviours in Manhattan's most dangerous intersections. Inj. Prev. 2014, 20, 343-346. [CrossRef] [PubMed]

5. Nasar, J.L.; Troyer, D. Pedestrian injuries due to mobile phone use in public places. Accid. Anal. Prev. 2013, 57, 91-95. [CrossRef] [PubMed]

6. National Transportation Safety Board. Preliminary Report Highway HWY18MH010; National Transportation Safety Board: Washington, DC, USA, 2018.

7. Dollar, P.; Wojek, C.; Schiele, B.; Perona, P. Pedestrian Detection: An Evaluation of the State of the Art. IEEE Trans. Pattern Anal. Mach. Intell. 2012, 34, 743-761. [CrossRef] [PubMed]

8. Dhondge, K.; Song, S.; Choi, B.-Y.; Park, H. WiFiHonk: Smartphone-Based Beacon Stuffed WiFi Car2X-Communication System for Vulnerable Road User Safety. In Proceedings of the 2014 IEEE 79th Vehicular Technology Conference (VTC Spring), Seoul, Korea, 18-21 May 2014; pp. 1-5. [CrossRef]

9. Wang, T.; Cardone, G.; Corradi, A.; Torresani, L.; Campbell, A.T. WalkSafe: A pedestrian safety app for mobile phone users who walk and talk while crossing roads. In Proceedings of the Twelfth Workshop on Mobile Computing Systems \& ApplicationsHotMobile '12, San Diego, CA, USA, 28-29 February 2012; pp. 1-6. [CrossRef]

10. Sarter, N.B. Multimodal information presentation: Design guidance and research challenges. Int. J. Ind. Ergon. 2006, 36, 439-445. [CrossRef]

11. Wang, M.; Liao, Y.; Lyckvi, S.L.; Chen, F. How drivers respond to visual vs. auditory information in advisory traffic information systems. Behav. Inf. Technol. 2019, 39, 1308-1319. [CrossRef]

12. Costanza, E.; Inverso, S.A.; Pavlov, E.; Allen, R.; Maes, P. eye-q: Eyeglass peripheral display for subtle intimate notifications. In Proceedings of the 8th Conference on Human-Computer Interaction with Mobile Devices and Services-MobileHCI '06, Helsinki, Finland, 12-15 September 2006; p. 211. [CrossRef]

13. Caudell, T.P.; Mizell, D.W. Augmented reality: An application of heads-up display technology to manual manufacturing processes. In Proceedings of the Twenty-Fifth Hawaii International Conference on System Sciences, Kauai, HI, USA, 7-10 January 1992; pp. 659-669. [CrossRef]

14. Carmigniani, J.; Furht, B.; Anisetti, M.; Ceravolo, P.; Damiani, E.; Ivkovic, M. Augmented reality technologies, systems and applications. Multimed. Tools Appl. 2011, 51,342-377. [CrossRef]

15. Henrysson, A.; Ollila, M.; Billinghurst, M. Mobile phone based AR scene assembly. In Proceedings of the 4th International Conference on Mobile and Ubiquitous Multimedia-MUM '05, Christchurch, New Zealand, 8-10 December 2005; p. 95. [CrossRef] 
16. Kim, H.; Wu, X.; Gabbard, J.L.; Polys, N.F. Exploring head-up augmented reality interfaces for crash warning systems. In Proceedings of the 5th International Conference on Automotive User Interfaces and Interactive Vehicular Applications-AutomotiveUI'13, Eindhoven, The Netherlands, 28-30 October 2013; pp. 224-227. [CrossRef]

17. Plavsic, M.; Bubb, H.; Duschl, M.; Tonnis, M.; Klinker, G. Ergonomic Design and Evaluation of Augmented Reality Based Cautionary Warnings for Driving Assistance in Urban Environments. Presented at the 17th World Congress on Ergonomics (International Ergonomics Association, IEA), Beijing, China, 9-14 August 2009.

18. Google. GLASS ENTERPRISE EDITION 2 Tech Specs. 22 July 2021. Available online: https:/ /www.google.com/glass/tech-specs/ (accessed on 26 June 2021).

19. Dünser, A.; Grasset, R.; Seichter, H.; Billinghurst, M. Applying HCI Principles to AR Systems Design. Presented at the 2nd International Workshop at the IEEE Virtual Reality 2007, Charlotte, NC, USA, 11 March 2007.

20. Boff, K.R.; Lincoln, J.E. Engineering Data Compendium: Human Perception and Performance; John Wiley and Sons: New York, NY, USA, 1986.

21. Gregory, R.L. The Intelligent Eye; Weidenfeld \& Nicolson: London, UK, 1970.

22. Galitz, W.O. The essential Guide to User Interface Design; Wiley: Indianapolis, IN, USA, 1997.

23. Clifton, I.G. Android user Interface Design: Turning Ideas and Sketches into Beautifully Designed Apps; Addison-Wesley: Upper Saddle River, NJ, USA, 2013.

24. Wogalter, M.S.; Conzola, V.C.; Smith-Jackson, T.L. Research-based guidelines for warning design and evaluation. Appl. Ergon. 2002, 33, 219-230. [CrossRef]

25. Wogalter, M.S.; Desaulniers, D.R.; Brelsford, J.W. Consumer Products: How are the Hazards Perceived? Proc. Hum. Factors Soc. Annu. Meet. 1987, 31, 615-619. [CrossRef]

26. Wogalter, M.S.; Godfrey, S.S.; Fontenelle, G.A.; Desaulniers, D.R.; Rothstein, P.R.; Laughery, K.R. Effectiveness of Warnings. Hum. Factors J. Hum. Factors Ergon. Soc. 1987, 29, 599-612. [CrossRef]

27. Tong, Y.; Jia, B. An Augmented-reality-based Warning Interface for Pedestrians: User Interface Design and Evaluation. Proc. Hum. Factors Ergon. Soc. Annu. Meet. 2019, 63, 1834-1838. [CrossRef]

28. Turner, A.; Zeller, M.; Cowley, E.; Bray, B. Hologram stability. Microsoft, 3 July 2018. Available online: https: / / docs.microsoft. $\mathrm{com} / \mathrm{en}-\mathrm{us} /$ windows / mixed-reality/hologram-stability (accessed on 26 June 2021).

29. Redert, A.; Hendriks, E.; Biemond, J. Correspondence estimation in image pairs. IEEE Signal Process. Mag. 1999, 16, 29-46. [CrossRef]

30. Maynard, P. Drawing Distinctions: The Varieties of Graphic Expression; Cornell Univ. Press: Ithaca, NY, USA, 2005.

31. Horaud, R.; Dornaika, F.; Lamiroy, B.; Christy, S. Object Pose: The Link between Weak Perspective, Paraperspective and Full Perspective. Int. J. Comput. Vis. 1997, 22, 173-189. [CrossRef]

32. Sonka, M.; Hlavac, V.; Boyle, R. Image Processing, Analysis, and Machine Vision, 2nd ed.; PWS Pub.: Pacific Grove, CA, USA, 1999.

33. Sony. Smarteyeglass-Sed-E1 Specifications. 2018. Available online: https://developer.sony.com/develop/smarteyeglass-sed-e1 / specifications (accessed on 26 June 2021). 